\title{
Histone Deacetylase 2 Cell Autonomously Suppresses Excitatory and Enhances Inhibitory Synaptic Function in CA1 Pyramidal Neurons
}

\author{
Jesse E. Hanson, ${ }^{\star}$ Lunbin Deng, ${ }^{\star}$ David H. Hackos, Shih-Ching Lo, Benjamin E. Lauffer, Pascal Steiner, and Qiang Zhou
} Department of Neuroscience, Genentech, South San Francisco, California 94080

Histone deacetylase 2 (HDAC2) negatively regulates excitatory synapse number and memory performance. However, whether HDAC2 regulation of excitatory synapses occurs in a cell-autonomous manner and whether HDAC2 regulates inhibitory synaptic functions are not well understood. To examine these aspects of HDAC2 function, we used sparse transfection of rat hippocampal slice cultures and whole-cell recordings in pyramidal neurons. HDAC2 knockdown (KD) in single postsynaptic pyramidal neurons enhanced, whereas HDAC2 overexpression (OE) reduced, excitatory synaptic transmission. Postsynaptic KD of HDAC2 also facilitated expression of longterm potentiation induced by subthreshold induction stimuli, without altering long-term depression. In contrast, HDAC2 KD reduced, whereas $\mathrm{HDAC} 2 \mathrm{OE}$ enhanced, inhibitory synaptic transmission. Alterations of postsynaptic $\mathrm{GABA}_{\mathrm{A}}$ receptors $\left(\mathrm{GABA}_{\mathrm{A}} \mathrm{Rs}\right)$ likely underlie the impact of HDAC2 on inhibitory transmission. Consistent with this, we observed reduced transcript and protein levels of the $\mathrm{GABA}_{\mathrm{A}} \mathrm{R}$ $\gamma 2$ subunit and reduced surface expression of the $\alpha 2$ subunit after HDAC2 KD. Furthermore, we observed a reduction in synaptic but not tonic $\mathrm{GABA}_{\mathrm{A}} \mathrm{R}$ currents by HDAC2 $\mathrm{KD}$, suggesting that $\mathrm{HDAC} 2$ selectively affects synaptic abundance of functional $\mathrm{GABA}_{\mathrm{A}} \mathrm{Rs}$. Immunostaining for postsynaptic $\mathrm{GABA}_{\mathrm{A}} \mathrm{Rs}$ confirmed that $\mathrm{HDAC} 2 \mathrm{KD}$ and $\mathrm{OE}$ can regulate the synaptic abundance of these receptors. Together, these results highlight a role for HDAC2 in suppressing synaptic excitation and enhancing synaptic inhibition of hippocampal neurons. Therefore, a shift in the balance of synaptic excitation versus inhibition favoring excitation could contribute to the beneficial effects of reducing HDAC2 function in wild-type mice or of inhibiting HDACs in models of cognitive impairment.

\section{Introduction}

Histone deacetylases (HDACs) are a family of enzymes that lead to condensed chromatin structure and decreased gene transcription (Strahl and Allis, 2000; Jenuwein and Allis, 2001) by decreasing histone acetylation. Pharmacological inhibition or genetic deletion of HDACs results in increased histone acetylation, relaxed chromatin structure, and increased transcription of sets of genes, which in turn can cause both increases and decreases in expression of other genes. There is also evidence that HDACs can act through non-histone and even cytoplasmic substrates (Buchwald et al., 2009; Ocker, 2010). Recently, HDAC regulation of histone acetylation has been shown to contribute to both normal cognition and cognitive impairments in various neurological disorders (Fischer et al., 2010). Pharmacological inhibition of HDACs can restore impaired memory in rodent models of Alz-

\footnotetext{
Received July 3, 2012; revised Feb. 1, 2013; accepted Feb. 10, 2013

Author contributions: J.E.H., L.D., D.H.H., S.-C.L., B.E.L., P.S., and Q.Z. designed research; J.E.H., L.D., D.H.H., S.-C.L., B.E.L., and Q.Z. performed research; J.E.H., L.D., D.H.H., B.E.L., and Q.Z. analyzed data; J.E.H., L.D., and Q.Z. wrote the paper.

*J.E.H. and L.D. contributed equally to this work.

We thank Morgan Sheng and Baris Bingol for comments and discussion on the content of this manuscript.

The authors declare no competing financial interests.

This article is freely available online through the $J$ Neurosci Author Open Choice option.

Correspondence should be addressed to Qiang Zhou, 1 DNA Way, MS 230B, South San Francisco, CA 94080.

E-mail:zhou.qiang@gene.com.

DOI:10.1523/JNEUROSCI.3162-12.2013

Copyright $\odot 2013$ the authors $\quad 0270-6474 / 13 / 335924-06 \$ 15.00 / 0$
}

heimer's disease (AD) (Ricobaraza et al., 2009, 2012; Kilgore et al., 2010; Govindarajan et al., 2011). Transgenic studies demonstrate that HDAC2 (but not HDAC1) overexpression (OE) specifically impairs synaptic function and memory performance, whereas HDAC2 knock-out mice exhibit increased levels of excitatory synaptic markers, facilitated induction of synaptic potentiation, and enhanced fear memory (Guan et al., 2009). Moreover, HDAC2 is upregulated in the brains of both $\mathrm{AD}$ patients and model mice, and knockdown (KD) of HDAC2 rescues deficits in synaptic plasticity and neurodegeneration-associated memory dysfunction in the AD model mice (Gräff et al., 2012). However, in the knock-out mouse and shRNA KD studies, prolonged HDAC2 reduction precluded examination of acute and cell-autonomous roles of HDAC2. In this study, we show that HDAC2 can function in a cell-autonomous manner to modulate both basal excitatory synaptic transmission and synaptic plasticity and can also regulate inhibitory synaptic function. In addition, we identified enhanced accumulation of synaptic $\mathrm{GABA}_{\mathrm{A}}$ receptors $\left(\mathrm{GABA}_{\mathrm{A}} \mathrm{Rs}\right)$ as a potential mechanism of HDAC2 regulation of synaptic inhibition. Together, these results point to a direct role for HDAC2 in controlling the balance of excitation versus inhibition, which may contribute to the pro-cognitive effects of reduced HDAC function.

\section{Materials and Methods}

Slice cultures. Hippocampi were dissected from 7- to 8-d-old male Sprague Dawley rats in minimum essential medium (MEM) with $15 \mathrm{~mm}$ 
HEPES and $10 \mathrm{~mm}$ Tris buffer. Four hundred micrometer slices were cultured on Millicell CM culture plate inserts (Millipore). The culture medium consisted of 50\% MEM, 25\% HBSS, and 25\% horse serum, with $12.5 \mathrm{mM}$ HEPES buffer and penicillin $(100 \mathrm{U} / \mathrm{ml}) /$ streptomycin $(100$ $\mu \mathrm{g} / \mathrm{ml}$ ) (all from Invitrogen). Cultures were maintained in $5 \% \mathrm{CO}_{2}$ at $37^{\circ} \mathrm{C}$. Slices were maintained in vitro for 1 week before transfection.

Assay of shRNA KD. HEK293 cells expressing FLAG-tagged HDAC1 or HDAC2 were cotransfected at a ratio of 1:3 to 1:20 with the individual shRNA constructs or scrambled shRNA sequence with no target in the genome as a control. KD efficiency of each shRNA was assessed by first normalizing measurements from bands corresponding to HDAC1 or HDAC2 to measurements of the loading control bands from the same sample ( $\beta$-actin or GAPDH) and then comparing the normalized measurements with the value with scrambled shRNA. All measurements were made by quantifying the total intensity of enhanced chemiluminescence signals captured by a CCD camera within its linear range using Quantity One software (Bio-Rad).

Slice culture transfection. For KD experiments, slices were transfected using the gene gun (Bio-Rad) with gold particles coated with $10 \mu \mathrm{g}$ of a pCAGGS-eGFP reporter plasmid (a gift from Dr. Robby Weimer, Genentech, South San Francisco, CA) and $25 \mu \mathrm{g}$ each of four distinct HuSH shRNA constructs (targeting sequences indicated) against either of the following all in HuSH plasmids (OriGene): (1) HDAC1: (A) ATCCGT AATGTCGCTCGGTGCTGGACTTA; (B) ACAGCGACGACTACATCA AGTTCTTGCGT; (C) AAGCCAACGCTGAGGAGATGACCAAGTAC; (D) GCCATCCTGGAACTGCTAAAGTATCACCA; or (2) HDAC2: (A) AGAAAGTGTGCTACTATTATGACGGTGAT; (B) GCTTGTGATGAA GAGTTCTCAGATTCTGA;(C)TGATGCTTGGCGGAGGTGGCTACA CAATC; (D) TCAAAGGTCACGCTAAATGTGTAGAAGTA.

For $\mathrm{OE}$ experiments, gold particles were coated with either $10 \mu \mathrm{g}$ of eGFP plasmid alone as a control or along with $100 \mu \mathrm{g}$ of HDAC2 cDNAs in pCAGGS expression plasmids. Electrophysiological measurements were conducted 3-4 d after transfection.

Electrophysiology. For whole-cell recordings, patch-clamp recordings were made in oxygenated artificial CSF containing the following (in mM): 127 $\mathrm{NaCl}, 2.5 \mathrm{KCl}, 1.3 \mathrm{MgSO}_{4}, 2.5 \mathrm{CaCl}_{2}, 1.25 \mathrm{Na}_{2} \mathrm{HPO}_{4}, 25 \mathrm{NaHCO}_{3}$, and 25 glucose. Patch pipette internal solution consisted of the following (in $\mathrm{mM}$ ): 20 Cs-methanesulfonate, $20 \mathrm{CsCl}$, 0.5 EGTA, 10 HEPES, $2.5 \mathrm{MgCl}_{2}, 4$ $\mathrm{Na}_{2}$ ATP, $0.3 \mathrm{Na}_{3} \mathrm{GTP}, 10$ phosphocreatine, and $5 \mathrm{~mm}$ QX-314. mEPSCs were recorded in the presence of picrotoxin (PTX) and tetrodotoxin (TTX) at $-70 \mathrm{mV}$ and mIPSCs in the presence of 2,3-dihydroxy-6-nitro-7sulfamoyl-benzo[f]quinoxaline-2,3-dione (NBQX), D-(-)-2-amino-5phosphonopentanoic acid (AP-5), and TTX at $0 \mathrm{mV}$. To measure tonic $\mathrm{GABA}_{\mathrm{A}} \mathrm{R}$-mediated currents, $30 \mu \mathrm{M}$ bicuculline was puffed onto the recorded neuron with a Picospritzer in the presence of TTX, NBQX, and AP-5 (all from Tocris Biosciences). Significance of differences between mean frequencies or amplitudes of mEPSCs or mIPSCs was assessed using a Student's $t$ test. Cumulative amplitude distributions were plotted at 10 percentile intervals using values interpolated from $1 \mathrm{pA}$ binned data for each cell. For synaptic plasticity experiments, evoked EPSCs were measured at $-70 \mathrm{mV}$ in the presence of PTX. In the subthreshold long-term potentiation (LTP) induction experiments, after a brief baseline $(\sim 5 \mathrm{~min}), 100$ stimuli at $2 \mathrm{~Hz}$ were delivered while the postsynaptic neuron was voltage clamped at $0 \mathrm{mV}$. Long-term depression (LTD) was induced using 600 stimuli at $2 \mathrm{~Hz}$ while the postsynaptic neuron was voltage clamped at $-40 \mathrm{mV}$.

Quantitative PCR analysis of inhibitory gene expression. Primary dissociated hippocampal neurons were prepared as described previously (Deng et al., 2011). The most effective HDAC2 shRNA sequence (HDAC2 shRNA-B) was expressed using a U6 promoter in an adenoassociated virus vector. Virus with a scrambled shRNA sequence was used as a control. Hippocampal cultures were infected at $11 \mathrm{~d}$ in vitro and collected for quantitative PCR (qPCR) analysis on 16-17 d in vitro. qPCR was performed with a Cells-to-CT Gene Expression kit (Applied Biosystems) following the instructions of the manufacturer.

Western blot analysis of total and surface $G A B A_{A} R$ subunits. Protein samples for assaying total and surface $G_{A B A} R$ subunits were prepared from brain homogenates. Surface $\mathrm{GABA}_{\mathrm{A}} \mathrm{R}$ biotinylation was performed with a bicinchoninic acid kit (Pierce Biotechnology) following the instructions of the manufacturer. Antibodies were used at the following dilutions: anti- $\alpha 1 \mathrm{GABA}_{\mathrm{A}} \mathrm{R}$ (1:1000; NeuroMab); anti- $\alpha 2 \mathrm{GABA}_{\mathrm{A}} \mathrm{R}$ (1: 1000 ; Alomone Labs); anti- $\gamma 2 \mathrm{GABA}_{\mathrm{A}} \mathrm{R}$ (1:1000; Alpha Diagnostic International); anti-transferrin receptor (1:2000; Invitrogen); anti- $\beta$-actin (1: 2000; Cell Signaling Technology); and anti-FLAG (1:4000; Sigma).

Immunostaining. Double immunostaining was performed as reported previously (Deng et al., 2011) with anti-GABA $\mathrm{A} \alpha 2$ subunit rabbit polyclonal (1:1000; SYSY) and anti-vesicular GABA transporter (vGAT) mouse monoclonal antibodies (1:1000; SYSY). Single sections were acquired using a confocal microscope with identical settings applied to all samples from all conditions in an experiment. $\mathrm{GABA}_{\mathrm{A}} \mathrm{R}$ synaptic abundance was measured as the total fluorescent intensity of $\alpha 2$-positive puncta that were colocalized with presynaptic vGAT staining. The experimenter was blind to the condition in all experiments.

\section{Results}

\section{HDAC2 suppresses basal excitatory synaptic transmission in a cell-autonomous manner}

To examine cell-autonomous impacts of altered HDAC levels on synaptic function, KD was achieved in organotypic slice cultures by biolistically transfecting individual CA1 pyramidal neurons with shRNA selectively targeting either HDAC2 (Fig. $1 A, B$ ) or HDAC1 (Fig. 1C,D). Cotransfection with GFP allowed targeting transfected neurons for whole-cell patch-clamp recordings. Only a few neurons were transfected in each slice, and untransfected neighboring neurons within the same slice were used as controls. Therefore, we consider phenotypes selective to the genetically manipulated cells to reflect cell-autonomous impacts. Compared with untransfected neurons, HDAC2 KD caused a significant increase in mEPSC amplitude without altering mEPSC frequency (Fig. 1E,F), whereas HDAC1 KD did not significantly alter mEPSC amplitude or frequency (Fig. $1 G, H$ ). These results suggest that HDAC2 but not HDAC1 regulates excitatory synaptic transmission. To further test that HDAC2 is responsible for the observed effect, we examined HDAC2 OE in single pyramidal neurons using the same biolistic transfection method. Reduced mEPSC amplitude but no change in mEPSC frequency was observed with HDAC2 OE (Fig. $1 I, J$ ), whereas GFP expression alone had no effect on mEPSCs (Fig. $1 K, L$ ). Together, the KD and $\mathrm{OE}$ results strongly support a cell-autonomous role for HDAC2 in regulating excitatory synaptic function in excitatory neurons.

\section{HDAC2 functions in postsynaptic neurons to limit potentiation of excitatory synapses}

To test whether HDAC2 reduction in a single postsynaptic pyramidal neuron is sufficient to affect plasticity of excitatory synapses, we examined LTP of evoked EPSCs recorded from transfected pyramidal neurons. Induction of LTP was attempted from HDAC2 or HDAC1 KD neurons by pairing high-frequency presynaptic stimulation with postsynaptic depolarization. A weak induction protocol that did not lead to LTP in either untransfected neurons ( $98 \pm 8.9 \%$ of baseline) or neurons transfected with scrambled shRNA ( $105 \pm 4.2 \%$ of baseline) was used. In HDAC2 KD neurons, robust LTP was observed using this normally subthreshold induction protocol (Fig. 2A). For comparison, the same induction protocol was applied to neurons with HDAC1 KD, because HDAC1 KD did not have any significant impacts on synaptic function in the previous experiments and HDAC1 transgenic mice exhibit normal synaptic and cognitive function (Guan et al., 2009). In contrast to HDAC2 KD neurons, no LTP was induced in HDAC1 KD neurons, confirming the selective enhancement of LTP by HDAC2 KD (Fig. 2A). We also examined the effect of HDAC2 KD on LTD using a protocol that induced robust LTD in untransfected neurons $(40.3 \pm 8.6 \%$ 
of baseline) and in neurons transfected with scrambled shRNA $(43.6 \pm 8.1 \%$ of baseline). We observed no difference in LTD between neurons with HDAC2 versus HDAC1 KD, with both exhibiting robust LTD (Fig. 2B). Together, these results suggest that HDAC2 functions within postsynaptic pyramidal neurons to limit LTP but do not suggest a significant role for HDAC2 in LTD.

\section{HDAC2 enhances synaptic inhibition in a cell-autonomous manner}

Although, to our knowledge, a role for HDAC2 in regulating inhibitory synaptic functions has not been described previously, we examined the impacts of manipulating HDAC2 levels on mIPSCs in neurons in slice culture. HDAC2 KD resulted in a significant decrease in both amplitude and frequency of mIPSCs compared with untransfected neighboring neurons (Fig. $3 A, B$ ), whereas HDAC1 KD did not significantly alter mIPSCs (Fig. $3 C, D)$. HDAC2 OE significantly increased the amplitude of mIPSCs without altering mIPSC frequency (Fig. $3 E, F$ ), whereas GFP expression alone had no effect on mIPSCs (Fig. 3G,H). Together, these results strongly support a cell-autonomous role for HDAC2 in enhancing inhibitory synaptic function and suggest that HDAC2 can regulate the abundance and/or function of synaptic $\mathrm{GABA}_{\mathrm{A}}$ Rs. Because the extremely sparse transfection achieved in slice cultures precludes evaluating this hypothesis using biochemical methods, we tested whether virusmediated KD of HDAC2 in primary neuronal cultures, which could provide the high transfection efficiency required, could recapitulate the phenotype of reduced inhibitory function. We first characterized the efficacy of viral HDAC2 $\mathrm{KD}$ in primary cultures and found dramatic decreases in both HDAC2 mRNA and protein levels, indicating that $\mathrm{KD}$ was achieved broadly throughout the cultures (Figs. 3I, 4A). As in the slice culture experiments, HDAC2 KD resulted in reduced mIPSC amplitude (Fig. $3 J$ ), but contrasting with the slice culture experiments, there was no change in MIPSC frequency (Fig. $3 K$ ). A possible explanation for this difference could be that presynaptic neurons are affected or network-wide compensation occurs in response to mass transfection, so as to mask the effects that occur in the cell-autonomous situation. To determine whether extrasynaptic $\mathrm{GABA}_{\mathrm{A}}$ Rs could also be affected, we measured tonic $\mathrm{GABA}_{\mathrm{A}} \mathrm{R}$ currents that are mediated by extrasynaptic receptors and
A

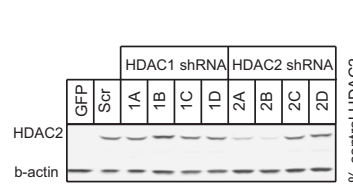

B

C

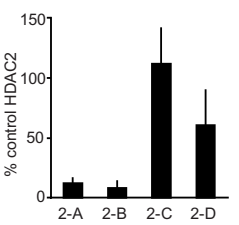

E

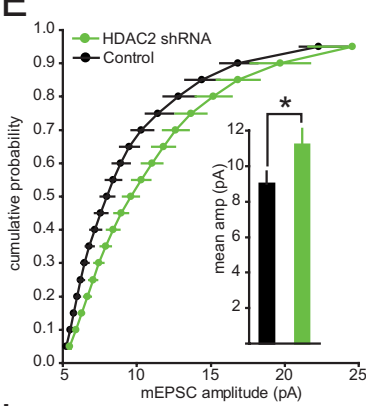

$\mathrm{F}$
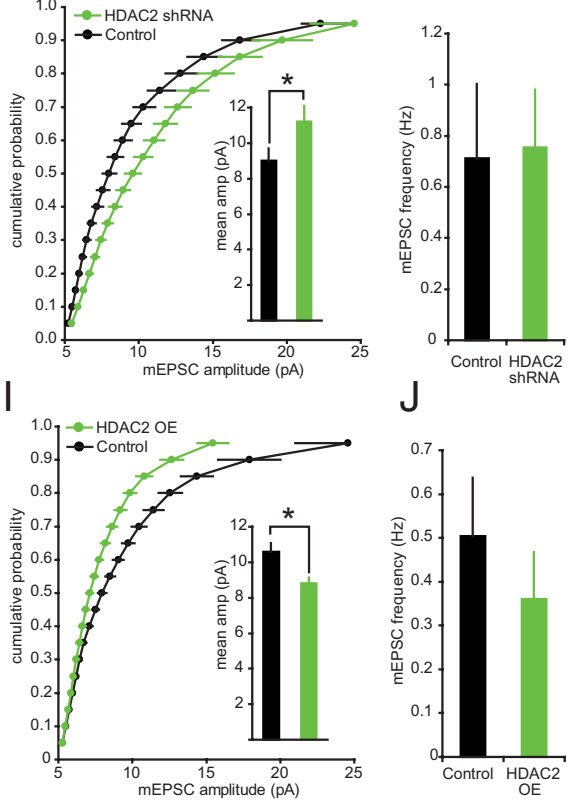

$J$

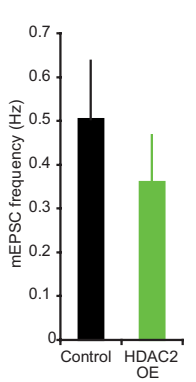

$\mathrm{K}$
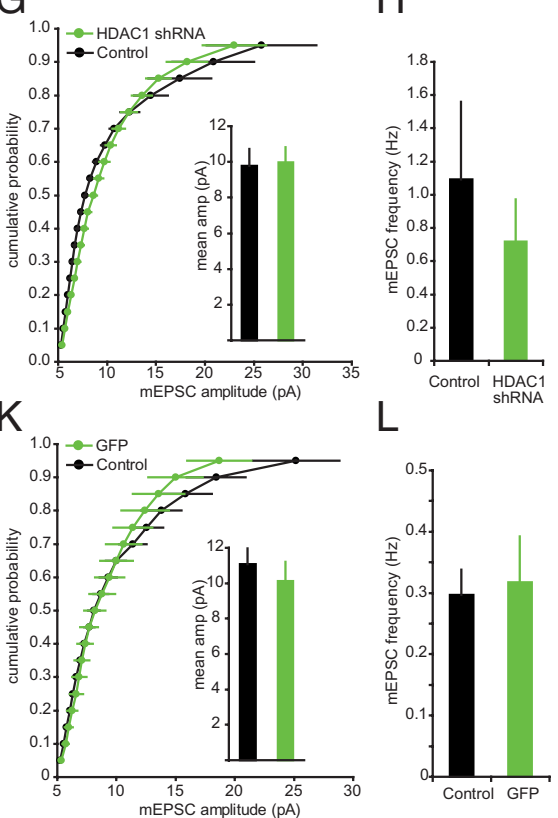

Figure 1. HDAC2 suppresses excitatory transmission. $\boldsymbol{A}$, Western blots of lysates from HEK293 cells expressing HDAC2 probed with anti-FLAG to assess HDAC2 expression or anti- $\beta$-actin as a loading control. Examples of KD with the four different HDAC2 shRNA or HDAC1 shRNA sequences are shown, as well as samples lacking HDAC2 (GFP) or with scrambled shRNA as a control. $\boldsymbol{B}$, Quantification of KD of HDAC2 protein by the four HDAC2 shRNAs $(n=5)$. C, Western blot analysis of HDAC1 KD with examples of KD using four different HDAC1 shRNA or HDAC2 shRNA sequences and samples lacking HDAC1 (GFP) or with scrambled shRNA as a control. D, Quantification of KD of HDAC1 protein by the four HDAC1 shRNAs $(n=5)$. $\boldsymbol{E}$, HDAC2 KD increased mEPSC amplitude compared with neighboring untransfected CA1 neurons, seen as a rightward shift of the cumulative amplitude distribution and a significantly increased mean mEPSC amplitude ( $p<0.05, n=9$ and 12$). F$, HDAC2 KD did not significantly alter mEPSC frequency ( $n=9$ and 12$)$. G, HDAC1 KD did not significantly alter mEPSC amplitude ( $n=10$ and 12 ). $\boldsymbol{H}$, HDAC1 KD did not significantly alter mEPSC frequency ( $n=10$ and 12). I, HDAC2 OE reduced mEPSC amplitude compared with neighboring untransfected CA1 neurons $(p<0.05, n=14$ and 14).J, HDAC2 OE did not significantly alter mEPSC frequency ( $n=14$ and 14$) . \boldsymbol{K}$, GFP expression alone did not significantly alter mEPSC amplitude ( $n=12$ and 11$)$. L, GFP expression alone did not significantly alter mEPSC frequency $(n=$ 12 and 11). All data are plotted as mean \pm SEM.

A

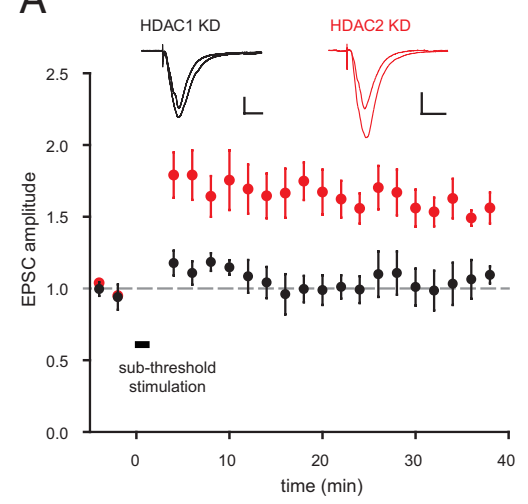

B

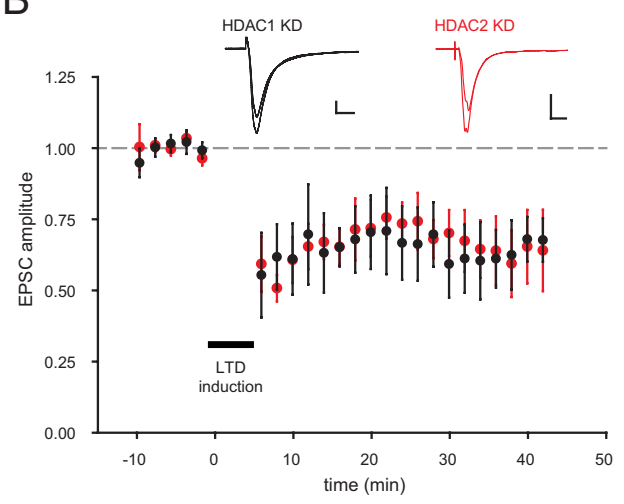

Figure 2. HDAC2 KD lowers the threshold for synaptic potentiation without affecting synaptic depression. $A$, Using a subthreshold induction protocol, no LTP was observed in neurons transfected with HDAC1 shRNA $(104 \pm 2 \%, n=8)$, whereas neurons transfected with HDAC2 shRNA exhibited robust LTP $(155 \pm 2 \%, n=12)$. Example EPSC traces before and after induction are shown inset. $\boldsymbol{B}$, Neurons transfected with HDAC1 shRNA or HDAC2 shRNA were equally capable of expressing robust LTD (HDAC1, $63 \pm 1 \%, n=6 ; H D A C 2,64 \pm$ $2 \%, n=10)$. Calibration: $50 \mathrm{pA}, 20 \mathrm{~ms}$. All data are plotted as mean \pm SEM. 
A

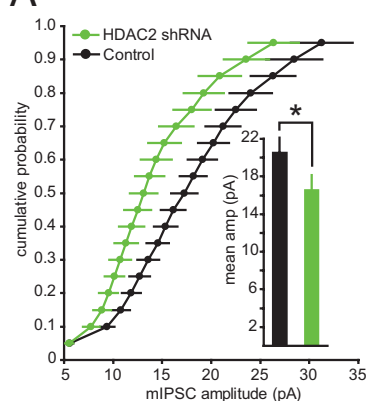

E

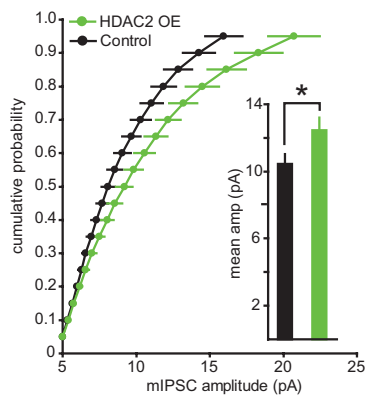

I

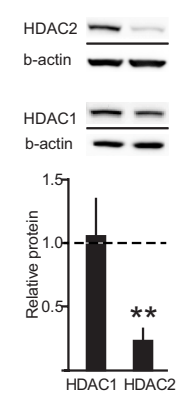

B

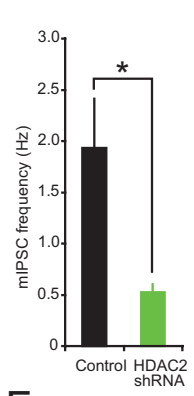

F

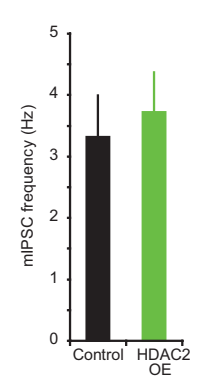

J
C

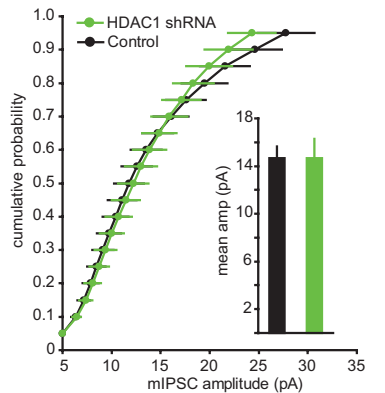

G
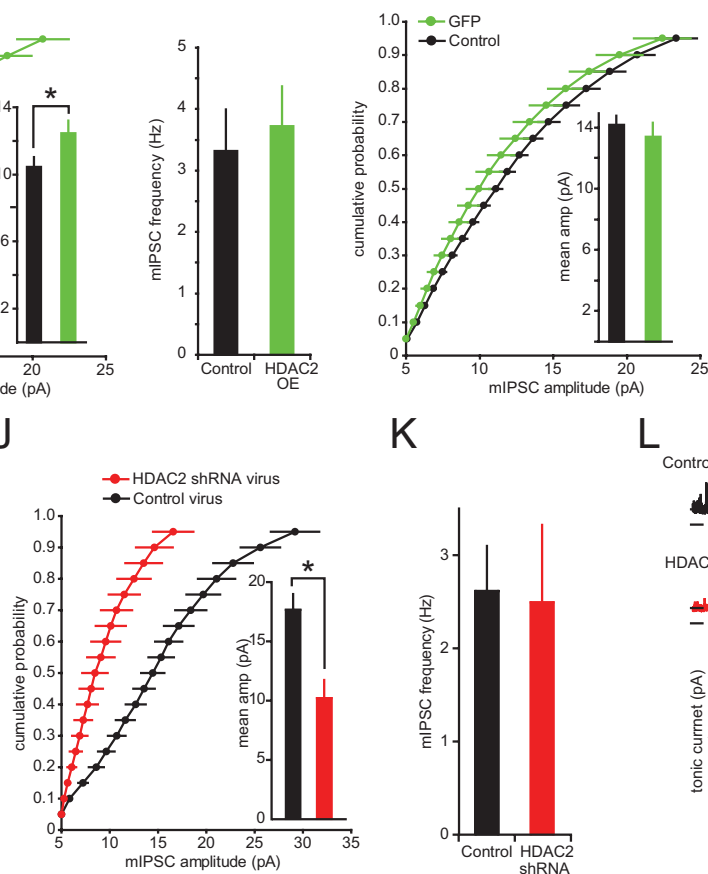

K

D

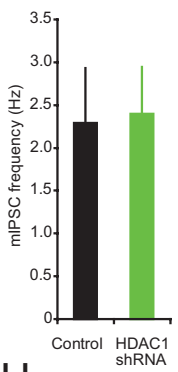

$\mathrm{H}$
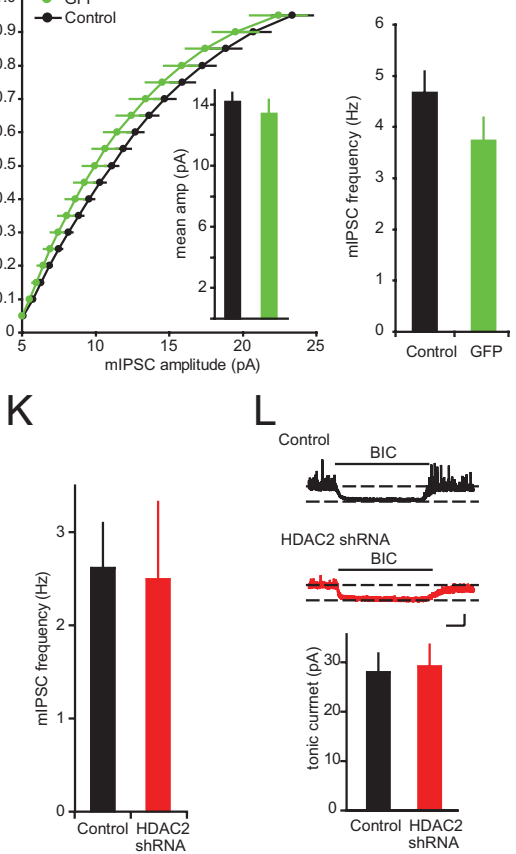

Figure 3. HDAC2 enhances inhibitory synaptic transmission. $A$, HDAC2 KD reduced mIPSC amplitude compared with neighboring untransfected CA1 neurons ( ${ }^{*} p<0.05, n=12$ and 15$)$. B, HDAC2 KD significantly decreased mean mIPSC frequency $\left({ }^{*} p<\right.$ $0.05, n=12$ and 15). C, HDAC1 KD did not significantly alter mIPSC amplitude ( $n=8$ and 7). D, HDAC1 KD did not significantly alter mIPSC frequency ( $n=8$ and 7). E, HDAC2 0 E enhanced mIPSC amplitude compared with neighboring untransfected CA1 neurons $\left({ }^{*} p<0.05, n=12\right.$ and 12). $\boldsymbol{F}$, HDAC2 0 E did not significantly alter mean mIPSC frequency $(p<0.05, n=12$ and 12). G, GFP expression alone did not significantly alter mIPSC amplitude ( $n=15$ and 12$)$. $\boldsymbol{H}$, GFP expression alone did not significantly alter mIPSC frequency ( $n=15$ and 12). I, Transfection of neuronal cultures using virus expressing HDAC2 shRNA resulted in a significant reduction in HDAC2 $(* * p<0.01, n=3)$ but not HDAC1 $(p>0.05, n=3)$ protein levels. J, Viral HDAC2 KD reduced mIPSC amplitude compared with cultures transfected with the control virus ( ${ }^{*} p<0.05, n=10$ and 7). $\boldsymbol{K}$, Viral HDAC2 KD did not significantly alter mIPSC frequency ( $n=7$ and 10). L, HDAC2 KD did not significantly alter tonic GABA $A_{A} R$ current ( $n=6$ and 7 ). Calibration: $50 \mathrm{pA}, 10 \mathrm{~s}$. All data are plotted as mean \pm SEM. BIC, Bicuculline.

found that the tonic $\mathrm{GABA}_{\mathrm{A}} \mathrm{R}$ currents were not altered by HDAC2 KD (Fig. $3 L$ ), indicating unaltered localization/function of extrasynaptic $\mathrm{GABA}_{\mathrm{A}} \mathrm{Rs}$ and selective alterations to synaptic $\mathrm{GABA}_{\mathrm{A}} \mathrm{Rs}$.

\section{HDAC2 regulates synaptic abundance of $\mathrm{GABA}_{\mathrm{A}} \mathrm{Rs}$}

To assess potential mechanisms by which HDAC2 selectively regulates synaptic $\mathrm{GABA}_{\mathrm{A}} \mathrm{R}$ function, we examined mRNA levels of several genes known to be involved in the assembly and synaptic targeting of $\mathrm{GABA}_{\mathrm{A}}$ Rs after broad HDAC2 KD in neuronal cultures. We observed a significant decrease in the mRNA levels of the $\mathrm{GABA}_{\mathrm{A}} \mathrm{R} \gamma 2$ subunit but not the other genes tested after HDAC2 KD (Fig. 4A). We also found a significant reduction in both total and surface levels of $\gamma 2$ subunits in neuronal cultures after HDAC2 KD (Fig. 4B). Although there was no reduction in

the total level of either $\alpha 1$ or $\alpha 2$ subunits, $\alpha 2$ but not $\alpha 1$ subunits exhibited significantly reduced surface levels after HDAC2 KD (Fig. 4B). Because $\gamma 2$ subunits have been implicated in synaptic targeting of $\mathrm{GABA}_{\mathrm{A}} \mathrm{Rs}$ and $\alpha 2$ subunits are the predominant $\alpha$ subunit at synapses on hippocampal pyramidal neurons (Essrich et al., 1998), our results could reflect reduced synaptic abundance of $\mathrm{GABA}_{\mathrm{A}} \mathrm{Rs}$ after HDAC2 KD. To obtain an independent measurement of synaptic $\mathrm{GABA}_{\mathrm{A}} \mathrm{Rs}$, we examined the $\alpha 2$ subunit using double immunostaining. Although the density of synapses and size of presynaptic terminals as measured by total intensity of vGAT puncta were unchanged, there was a clear reduction in the total intensity of $\mathrm{GABA}_{\mathrm{A}} \mathrm{R} \alpha 2$ subunit puncta that were colocalized with vGAT puncta in HDAC2 KD cultures (Fig. 4C), indicating a reduction in the abundance of synaptic $\mathrm{GABA}_{\mathrm{A}}$ Rs. $\mathrm{GABA}_{\mathrm{A}} \mathrm{R} \alpha 1$ subunit puncta intensity was unchanged (control, $43.8 \pm$ 5.8; KD, $47.3 \pm 3.8 ; n=3$ ), consistent with the unaltered total and surface levels of this subunit after HDAC2 KD.

To facilitate comparison with the sparsely transfected slice cultures, we also examined HDAC2 KD using Lipofectamine-mediated sparse transfection of neuronal cultures. As with broad viral infection, we observed a reduction in postsynaptic $\mathrm{GABA}_{\mathrm{A}} \mathrm{R}$ intensity that was already detectable $2 \mathrm{~d}$ after transfection (Fig. 4D). Although the density of presynaptic and postsynaptic markers was unaltered $2 \mathrm{~d}$ after $\mathrm{KD}$, after $4 \mathrm{~d}$ there was a significant decrease in the density of GABAergic synapses onto transfected neurons, suggesting that postsynaptic weakening was followed by synapse loss. When attempting to examine the effects of HDAC2 OE, we observed significant cell death when HDAC2 expressing virus was used to achieve broad transfection. Therefore, we performed immunostaining using sparse HDAC2 OE, which did not cause cell death. These experiments showed that, converse to $\mathrm{KD}, \mathrm{HDAC} 2 \mathrm{OE}$ led to an increase in synaptic $\mathrm{GABA}_{\mathrm{A}} \mathrm{R}$ staining intensity (Fig. 4E). Although the density of presynaptic markers was unchanged, there was an increase in dendritic $G_{A B A} R$ puncta density, suggesting aberrant accumulation of $\mathrm{GABA}_{\mathrm{A}} \mathrm{Rs}$ at nonsynaptic sites after HDAC2 OE. Together, these results support that HDAC2 affects inhibitory synaptic transmission by regulating synaptic abundance of $\mathrm{GABA}_{\mathrm{A}} \mathrm{Rs}$.

\section{Discussion}

Our results demonstrate that HDAC2 functions cell autonomously to regulate excitatory and inhibitory synaptic transmission in an opposite manner; HDAC2 suppresses basal transmission and potentiation of excitatory synapses and enhances inhibitory synaptic transmission, with the latter via mech- 
A

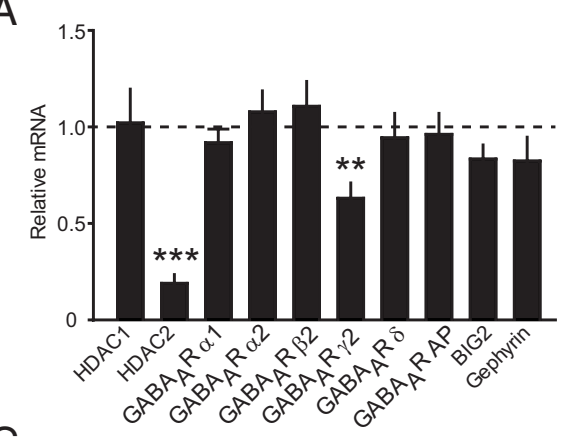

C

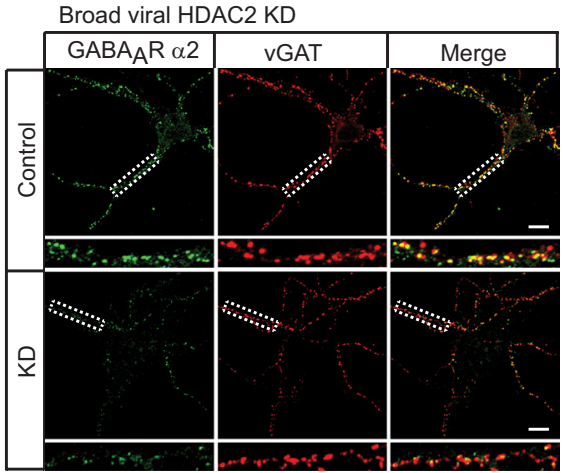

B
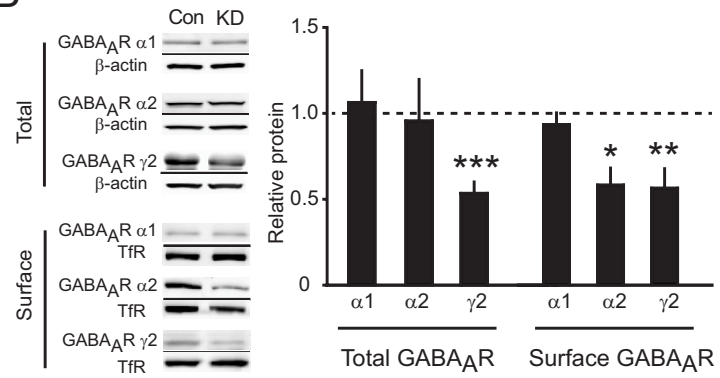
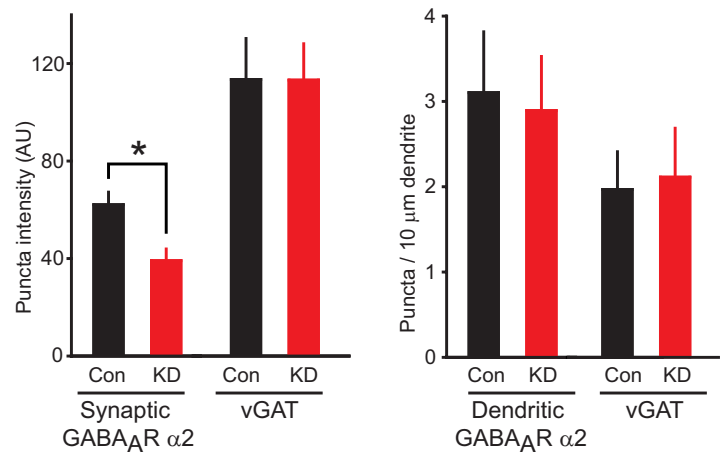

D
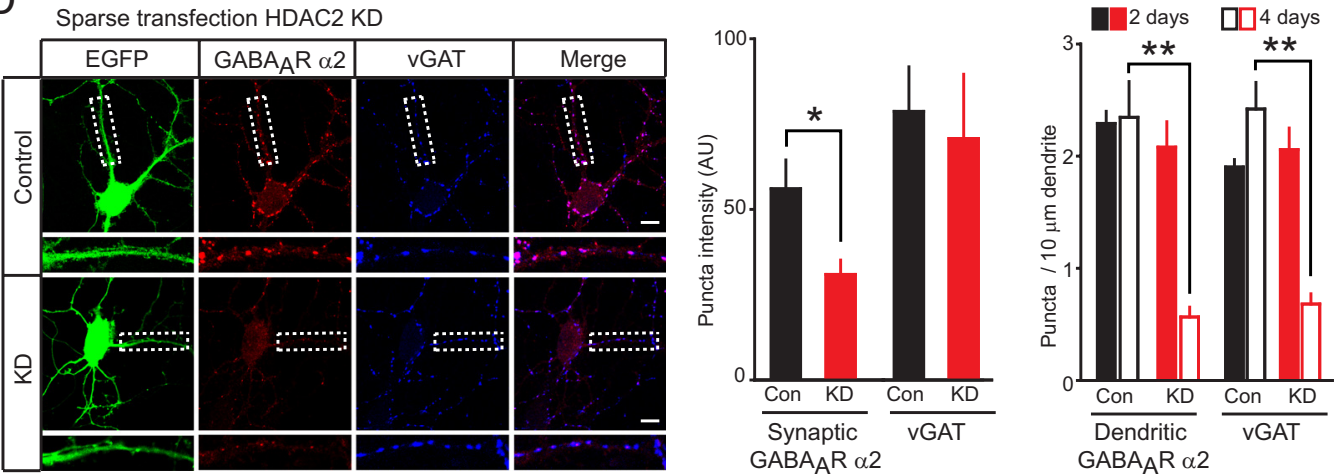

$\mathrm{E}$

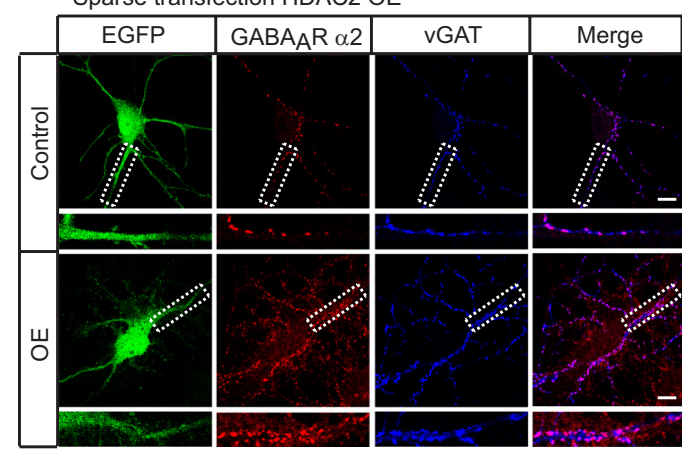

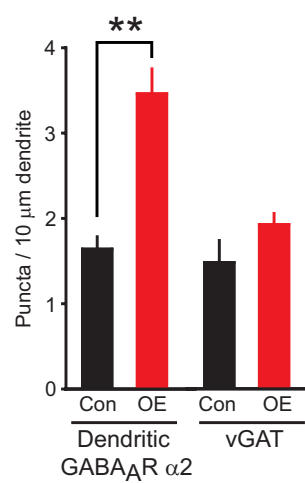

Figure 4. $H D A C 2$ regulates synaptic abundance of $G A B A_{A} R s$. $A$, Viral-mediated $H D A C 2$ shRNA expression in primary neuronal cultures resulted in a strong reduction of $H D A C 2$ but not $H D A C 1 \mathrm{mRNA}$ levels $(p<0.001, n=3)$. HDAC2 KD also resulted in a significant decrease in the $\mathrm{GABA}_{A} \mathrm{R} \gamma 2$ subunit mRNA levels $(p<0.01, n=3)$ but not the $\mathrm{GABA}_{A} \mathrm{R} \alpha 1, \alpha 2, \beta 2$, or $\delta$ subunits, GABA $A_{A} \mathrm{RAP}$ (adaptor protein), BIG2 (brefeldin A-inhibited GDP/GTP exchange factor 2), or Gephyrin. $B$, HDAC2 KD resulted in reduced total and surface expression of the $\gamma 2$ subunit ( $p<0.001$ and 0.01 , respectively) and reduced surface expression of the $\alpha 2$ subunit $(p<0.05)$ but not the total $\alpha 2$ subunit expression or total or surface expression of the $\alpha 1$ subunit $(n=3-4)$. C, Broad HDAC2 KD using viral transfection reduced the abundance of synaptic GABA $A_{A} R \alpha 2$ subunit labeling (green, $\mathrm{GABA}_{A} R \alpha 2$; red, $\mathrm{vGAT}$ ). The total fluorescence intensity of $\alpha 2$ subunit puncta that were colocalized with vGAT staining was significantly reduced $(p<0.05, n=$ 3), whereas the density of $\alpha 2$ puncta and the intensity and density of vGAT staining were not significantly altered. $\boldsymbol{D}$, Sparse HDAC2 KD significantly reduced the intensity of synaptic $\alpha 2$ subunit staining in transfected neurons identified by GFP expression $(p<0.05, n=3)$, whereas the intensity of VGAT staining was not significantly altered. The density of $\alpha 2$ subunit and vGAT puncta were significantly reduced at $4 \mathrm{~d}$ but not $2 \mathrm{~d}$ after transfection $(p<0.01, n=3)$. E, Sparse HDAC2 OE resulted in significantly increased intensity of synaptic $\alpha 2$ subunit but not vGAT staining $(p<0.05 n=3)$. The density of $\alpha 2$ subunit but not vGAT puncta was significantly increased $(p<0.01 n=3)$. Scale bars, $10 \mu \mathrm{m}$. All data are plotted as mean \pm SEM. Con, Control. ${ }^{*} p<0.05,{ }^{* *} p<0.01,{ }^{* * *} p<0.001$. 
anisms likely involving regulation of the synaptic abundance of $\mathrm{GABA}_{\mathrm{A}}$ Rs. These results provide several novel insights into HDAC2 regulation of synaptic transmission, plasticity, and the balance between excitation and inhibition.

Our observation that HDAC2 KD can increase mEPSC amplitude in slice cultures is consistent with the increased acetylation of the promoter regions of many genes involved in excitatory synaptic function in HDAC2 knock-out mice (Guan et al., 2009). Consistent with this, we were able to detect increased surface expression of AMPA receptor subunit GluA2 (128.6 $\pm 9.4 \%$ in control, $p<0.05$, $n=3$ ) after HDAC2 KD. Contrasting with our results, a previous study using cre recombinase to broadly delete HDAC2 during in vitro development of dissociated neurons did not observe enhanced mEPSC amplitude but rather a decrease in mEPSC frequency under some conditions (Akhtar et al., 2009). These previous results likely speak to non-cell-autonomous impacts specific to the experimental preparation, because the effects of HDAC2 deletion differed depending on the developmental stage of the cultures, and phenotypes were lost when infection rate was reduced from $>80$ to $<20 \%$. In contrast, our mEPSC results speak to the cell-autonomous effects of HDAC2 KD attributable to the very low $(<0.1 \%)$ transfection rate, and the use of neighboring untransfected neurons as controls rules out effects dependent on the presynaptic environment. Although our slice culture preparation preserves the trisynaptic circuit of the hippocampus (Gutiérrez and Heinemann, 1999) and allows examination of pyramidal neurons in a more in vivo-like context, ultimately in vivo studies using sparse transfection will better speak to the proximal functional impacts of reducing HDAC2 in the brain.

Another novel finding is that HDAC2 enhances inhibitory synaptic transmission in a cell-autonomous manner. The decreased mIPSC amplitude is consistent with the reduced abundance of postsynaptic $\mathrm{GABA}_{\mathrm{A}}$ Rs seen with immunostaining after HDAC2 KD. The reduction in mIPSC frequency with HDAC2 KD in slice cultures could be caused by reduced presynaptic release probability or reduced density of presynaptic terminals. The latter interpretation is supported by the reduced presynaptic marker density that followed reduced postsynaptic $\mathrm{GABA}_{\mathrm{A}} \mathrm{R}$ staining with sparse HDAC2 $\mathrm{KD}$, suggesting that synapse loss could be secondary to loss of postsynaptic receptors, as has been reported to occur after manipulations that reduce postsynaptic GABA $\mathrm{A}_{\mathrm{A}}$ (Li et al., 2005; Fang et al., 2006). The converse experiments showed that HDAC2 OE led to increased mIPSC amplitude and intensity of postsynaptic $\mathrm{GABA}_{\mathrm{A}} \mathrm{R}$ staining without altering synapse density. The regulation of inhibitory transmission was selective to synaptic $\mathrm{GABA}_{\mathrm{A}} \mathrm{R}$ currents, because tonic $\mathrm{GABA}_{\mathrm{A}} \mathrm{R}$ currents were unaffected by $\mathrm{HDAC} 2 \mathrm{KD}$, suggesting that HDAC2 does not regulate extrasynaptic $\mathrm{GABA}_{\mathrm{A}} \mathrm{R}$ localization/function. Consistent with an impact on synaptic $\mathrm{GABA}_{\mathrm{A}} \mathrm{Rs}$, we observed a decrease in $\mathrm{GABA}_{\mathrm{A}} \mathrm{R} \gamma 2$ subunit transcripts and protein level after HDAC2 $\mathrm{KD}$ in neuronal cultures and a reduced surface expression of the $\mathrm{GABA}_{\mathrm{A}} \mathrm{R} \alpha 2$ subunit. Although the tonic $\mathrm{GABA}_{\mathrm{A}} \mathrm{R}$ current is predominately mediated by extrasynaptic $\mathrm{GABA}_{\mathrm{A}}$ Rs usually composed of $\alpha 4 / 5, \beta$, and $\delta$ subunits, the majority of synaptic $\mathrm{GABA}_{\mathrm{A}} \mathrm{Rs}$ are composed of $\alpha 1 / 2, \beta$, and $\gamma 2$ subunits (Jacob et al., 2008). Although $\alpha$ and $\beta$ subunits are required for efficient assembly and surface expression of $\mathrm{GABA}_{\mathrm{A}} \mathrm{Rs}, \gamma 2$ subunits play a pivotal role in the synaptic targeting of $\mathrm{GABA}_{\mathrm{A}} \mathrm{Rs}$ (Essrich et al., 1998). Consistent with this, we observed reduced synaptic $\mathrm{GABA}_{\mathrm{A}} \mathrm{Rs}$ as detected by $\alpha 2$ subunit staining after HDAC2 KD. This decreased inhibitory synaptic function likely occurs as a consequence of increased transcription of certain sets of genes, which in turn leads to negative regulation of inhibitory synapses.

Together, our results suggest that suppressing HDAC2 function results in a coordinated adjustment in the balance of excit- atory versus inhibitory synaptic inputs onto CA1 pyramidal neurons, enhancing excitation and reducing inhibition. These effects, which would both act to favor neuronal activation, could in part mediate the pro-cognitive effects of genetic HDAC2 deletion (Guan et al., 2009) or of HDAC inhibition in mouse models of cognitive impairment (Ricobaraza et al., 2009, 2012; Kilgore et al., 2010; Govindarajan et al., 2011). Thus, epigenetic regulation of the balance between synaptic excitation and inhibition is an important aspect of HDAC2 function that should be considered in the context of pathological changes to, or attempted therapeutic modulation of, HDAC2.

\section{References}

Akhtar MW, Raingo J, Nelson ED, Montgomery RL, Olson EN, Kavalali ET, Monteggia LM (2009) Histone deacetylases 1 and 2 form a developmental switch that controls excitatory synapse maturation and function. J Neurosci 29:8288-8297. CrossRef Medline

Buchwald M, Krämer OH, Heinzel T (2009) HDACi: targets beyond chromatin. Cancer Lett 280:160-167. CrossRef Medline

Deng L, Kaeser PS, Xu W, Südhof TC (2011) RIM proteins activate vesicle priming by reversing autoinhibitory homodimerization of Munc13. Neuron 69:317-331. CrossRef Medline

Essrich C, Lorez M, Benson JA, Fritschy JM, Lüscher B (1998) Postsynaptic clustering of major GABAA receptor subtypes requires the gamma 2 subunit and gephyrin. Nat Neurosci 1:563-571. CrossRef Medline

Fang C, Deng L, Keller CA, Fukata M, Fukata Y, Chen G, Lüscher B (2006) GODZ-mediated palmitoylation of $\mathrm{GABA}_{\mathrm{A}}$ receptors is required for normal assembly and function of GABAergic inhibitory synapses. J Neurosci 26:12758-12768. CrossRef Medline

Fischer A, Sananbenesi F, Mungenast A, Tsai LH (2010) Targeting the correct HDAC(s) to treat cognitive disorders. Trends Pharmacol Sci 31:605617. CrossRef Medline

Govindarajan N, Agis-Balboa RC, Walter J, Sananbenesi F, Fischer A (2011) Sodium butyrate improves memory function in an Alzheimer's disease mouse model when administered at an advanced stage of disease progression. J Alzheimers Dis 26:187-197. CrossRef Medline

Gräff J, Rei D, Guan JS, Wang WY, Seo J, Hennig KM, Nieland TJ, Fass DM, Kao PF, Kahn M, Su SC, Samiei A, Joseph N, Haggarty SJ, Delalle I, Tsai LH (2012) An epigenetic blockade of cognitive functions in the neurodegenerating brain. Nature 483:222-226. CrossRef Medline

Guan JS, Haggarty SJ, Giacometti E, Dannenberg JH, Joseph N, Gao J, Nieland TJ, Zhou Y, Wang X, Mazitschek R, Bradner JE, DePinho RA, Jaenisch R, Tsai LH (2009) HDAC2 negatively regulates memory formation and synaptic plasticity. Nature 459:55-60. CrossRef Medline

Gutiérrez R, Heinemann U (1999) Synaptic reorganization in explanted cultures of rat hippocampus. Brain Res 815:304-316. CrossRef Medline

Jacob TC, Moss SJ, Jurd R (2008) GABA(A) receptor trafficking and its role in the dynamic modulation of neuronal inhibition. Nat Rev Neurosci 9:331-343. CrossRef Medline

Jenuwein T, Allis CD (2001) Translating the histone code. Science 293: 1074-1080. CrossRef Medline

Kilgore M, Miller CA, Fass DM, Hennig KM, Haggarty SJ, Sweatt JD, Rumbaugh G (2010) Inhibitors of class 1 histone deacetylases reverse contextual memory deficits in a mouse model of Alzheimer's disease. Neuropsychopharmacology 35:870-880. CrossRef Medline

Li RW, Yu W, Christie S, Miralles CP, Bai J, Loturco JJ, De Blas AL (2005) Disruption of postsynaptic GABA receptor clusters leads to decreased GABAergic innervation of pyramidal neurons. J Neurochem 95:756-770. CrossRef Medline

Ocker M (2010) Deacetylase inhibitors: focus on non-histone targets and effects. World J Biol Chem 1:55-61. CrossRef Medline

Ricobaraza A, Cuadrado-Tejedor M, Pérez-Mediavilla A, Frechilla D, Del Río J, García-Osta A (2009) Phenylbutyrate ameliorates cognitive deficit and reduces tau pathology in an Alzheimer's disease mouse model. Neuropsychopharmacology 34:1721-1732. CrossRef Medline

Ricobaraza A, Cuadrado-Tejedor M, Marco S, Pérez-Otaño I, García-Osta A (2012) Phenylbutyrate rescues dendritic spine loss associated with memory deficits in a mouse model of Alzheimer disease. Hippocampus 22: 1040-1050. CrossRef Medline

Strahl BD, Allis CD (2000) The language of covalent histone modifications. Nature 403:41-45. CrossRef Medline 